\title{
Safety Analysis of Reduced Route Spacing for RNP 2 under Radar Environment*
}

\author{
Ryota $\mathrm{MORI}^{\dagger}$ \\ Electronic Navigation Research Institute, Chofu, Tokyo 182-0012, Japan
}

\begin{abstract}
This paper conducts a safety assessment for reduced route spacing for RNP 2 aircraft under a radar environment. Although the criteria for $15 \mathrm{NM}$ separation standards exist, past safety assessment did not consider the surveillance environment. This consideration may reduce the possible route spacing. Here, to account for the surveillance environment, the recently developed ASEPS model is applied. Since this model was intended for deployment on oceanic route systems, the model parameters are modified appropriately while keeping the consistency of the past safety analysis and data analysis. In particular, the parameter of occupancy is set based on one-year flight data in Japanese airspace, and the calculation of action time to resolve the conflict is modified to estimate the collision probability more accurately. The results show that $8 \mathrm{NM}$ route spacing satisfies the safety criteria.
\end{abstract}

Key Words: Collision Risk Model, Safety, Collision Probability, Mid-air Collision, Performance-based Navigation

\section{Introduction}

Increasing air traffic requires more efficient use of airspace, and the performance-based navigation (PBN) concept has enabled reduced separation. In the PBN concept, as long as the aircraft is certified to certain navigation specifications (i.e., nav specs), it can fly on a designated route regardless of the type of navigation sensors. ${ }^{1)}$ There are various types of nav specs defined in the current PBN system, as shown in Fig. 1. Generally, there are two types of navigation specifications: RNAV and RNP. The main difference is that RNP requires on-board performance monitoring and an alerting function that alerts with more than $99.999 \%\left(=1-10^{-5}\right)$ probability when the aircraft deviates by more than double the RNP accuracy values, while RNAV does not impose this requirement. Since there is no protection of deviation in the RNAV system, RNAV has been mainly used under radar coverage. For both RNP and RNAV specifications, there are several nav specs, as shown in Fig. 1. Each nav spec is intended to be used for a certain flight phase. For example, RNP 4 is intended to be used for oceanic and remote airspaces only, and it cannot be used for en-route and terminal phases. The value of 4 indicates the RNP accuracy value, so the alert should be given if the aircraft may deviate by more than $8 \mathrm{NM}$ with the given probability.

The criteria of route spacing are given in several documents. PANS-ATM ${ }^{2)}$ is the main document, but some criteria are noted in the PBN manual ${ }^{1)}$ as well. In Japan, RNAV 5 has been introduced widely for en-route operations under radar coverage. According to the PBN manual, ${ }^{1)}$ up to $10-\mathrm{NM}$ spacing is acceptable if the resultant intervention rate is possible. As a result, 10-NM route spacing has been introduced

(C) 2021 The Japan Society for Aeronautical and Space Sciences

*Received 7 June 2020; final revision received 21 August 2020; accepted for publication 16 October 2020.

†Corresponding author, r-mori@mpat.go.jp for RNAV 5 routes in Japan. Japan also intends to replace RNAV 5 with RNP 2 for en-route operations. However, according to the current criteria in PANS-ATM, up to 15-NM separation is acceptable for RNP 2 en-route provided VHF communication is possible. This means that the route spacing needs to be set larger for RNP 2 to be introduced. However, the current PANS-ATM criteria do not consider the radar environment for the 15-NM separation standard. Accordingly, further safety analysis may require smaller separation than the current criteria. This paper investigates the potential of reduced route spacing of parallel routes by introducing RNP 2 to en-route operations. This RNP 2 en-route operation is called "proposed RNP2 operation" here.

When the separation standard or route spacing criteria are determined, numerical safety analysis is usually conducted. A collision risk model (CRM) is developed to calculate the collision probability under the considered operation, and the new standard can be introduced if the calculated collision probability is proven to be below the target level of safety (TLS). The value of TLS is determined in each regional airspace, ${ }^{3)}$ and the value of $5.0 \times 10^{-9}$ [fatal accident/flight hour] is widely used for lateral separation. As for the CRM, the Reich model ${ }^{4,5)}$ is the first, and the most popular, model to determine the lateral separation standard, where the collision probability is calculated using the probability density function of the lateral deviation. This model is simple but powerful, so most separation standards, including recent ones, ${ }^{6,7)}$ use this as a basis. In recent works, a new time-dependent model (i.e., Advanced Surveillance-enhanced Procedural Separation, ASEPS) for lateral separation has been developed, ${ }^{8,9)}$ and the Reich model is proven to show larger collision probability than the ASEPS model. This ASEPS model is intended for the future space-based ADS-B environment ${ }^{10)}$ over oceanic airspace, so it needs some modifications before being applied to the proposed RNP2 operation. Therefore, this paper reviews the existing CRMs first, and makes 


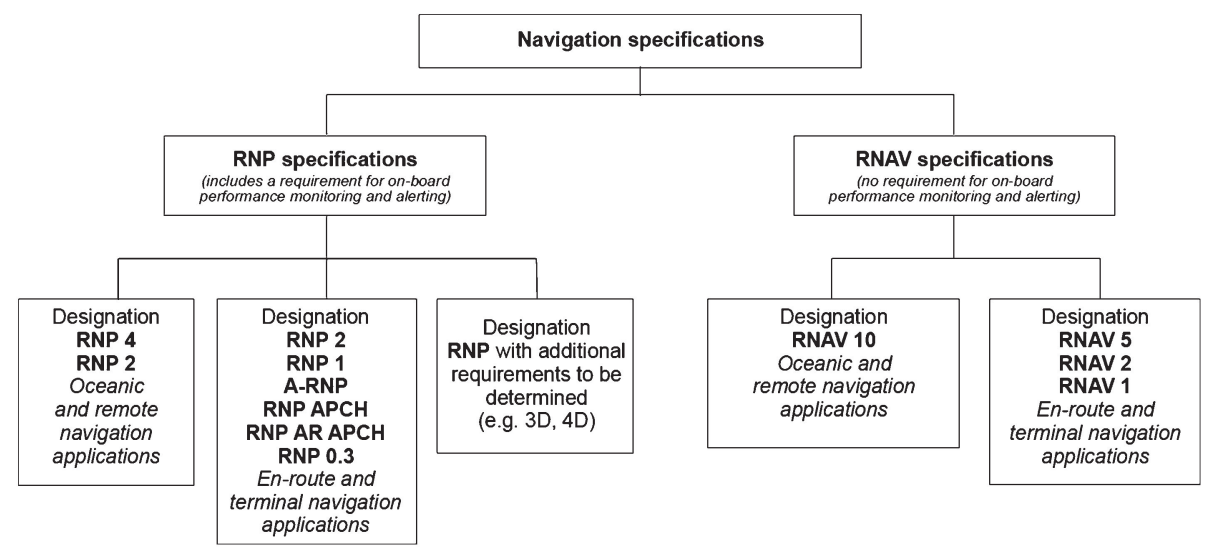

Fig. 1. Current navigation specifications. (Figure I-A-1-3 in Ref. 1))

required modifications to develop a new lateral separation standard for the proposed RNP2 operation.

\section{Review of Existing Models}

\subsection{Models to be considered}

To calculate the collision probability of two aircraft flying on parallel routes, there are two relevant mathematical models (CRMs): the Reich model and ASEPS model. The current criteria of both, 10-NM separation for RNAV 5 aircraft and 15-NM separation for RNP 2 aircraft, are determined using the Reich model. The Reich model has also been used to derive 50-, 30- and 23-NM lateral separation standards for parallel routes in an oceanic environment. Therefore, first, the derivation of 15-NM separation for RNP 2 aircraft is briefly reviewed, and the ASEPS model is discussed.

\subsection{Reich model for 15-NM separation of RNP 2 air- craft}

The derivation of this standard is summarized in Ref. 11), and more details are described in Guidelines for the Implementation of Lateral Separation Minima. ${ }^{7)}$ In this section, this derivation is briefly reviewed. This safety analysis is called 15-NM safety analysis here.

In the Reich model, the aircraft is modeled as a box, and the collision probability of 3-dimensional overlap of two boxes is calculated using the following formula:

$$
P=N_{x} P_{y}\left(S_{y}\right) P_{z}(0)\left(1+\frac{\lambda_{x}}{\lambda_{y}} \frac{\dot{y}}{\Delta V}+\frac{\lambda_{x}}{\lambda_{z}} \frac{\dot{z}}{\Delta V}\right)
$$

$N_{x}$ indicates the passing frequency; that is, the number of longitudinal overlaps per flight hour on two parallel routes. $P_{y}(y)$ indicates the lateral overlap probability when the nominal lateral separation is $y . P_{z}(0)$ indicates the vertical overlap probability when two aircraft are flying on the same flight level (i.e., 0 indicates the difference of the intended altitude). $\lambda_{x}, \lambda_{y}$, and $\lambda_{z}$ are the longitudinal, lateral, and vertical average aircraft size, respectively. $\Delta V$ is the relative speed of the two aircraft. $\dot{y}$ and $\dot{z}$ indicate the mean absolute relative lateral and vertical speed at the time of passing. The Reich model assumes three directional collisions, and the sum of three directional collisions is the total collision probability.
The key aspect of this model that needs to be considered is how to calculate the lateral overlap probability $P_{y}\left(S_{y}\right)$. The probability density function (PDF) of the lateral deviation of aircraft is defined as $f(y)$ (lateral-PDF), and the lateral overlap probability can be calculated using the following formula:

$$
P_{y}\left(S_{y}\right)=\int_{S_{y}-\lambda_{y}}^{S_{y}+\lambda_{y}} \int_{-\infty}^{\infty} f(y) f(z-y) d z d y
$$

To obtain the lateral-PDF, several deviation factors are considered, and the lateral-PDF caused by each factor is assumed. In the 15-NM safety analysis, the following four factors are considered.

\section{1) GNSS navigation error}

RNP 2 aircraft must use GNSS as the navigation system; so here, the maximum largest deviation of GNSS under normal conditions is assumed. The lateral-PDF by GNSS navigation error follows normal distribution with a standard deviation (SD) of $\sigma_{1}=0.5381 \mathrm{NM}$.

2) Navigation error during a RAIM outage

Receiver autonomous integrity monitoring (RAIM) is the function of GNSS to monitor integrity. In the Guidelines for the Implementation of Lateral Separation Minima, ${ }^{7)}$ the RAIM outage is assumed with a probability of $b=$ $1.9 \times 10^{-5}$. This value is obtained assuming 10 minutes for one-year flight $10 /(60 \times 24 \times 365)=1.9 \times 10^{-5}$. Under RAIM outage, the aircraft can follow the route for the first $2 \mathrm{~min}$, and starts deviating at 9 deg of track angle with a 95\% probability. However, the deviation will end within 5 min after RAIM outage due to ATC instructions. Finally, the maximum deviation at $5 \mathrm{~min}$ after RAIM outage is assumed, and the lateral-PDF due to RAIM outage is assumed to follow normal distribution with a SD of $\sigma_{2}=2.94 \mathrm{NM}$.

3) Flight technical error

Even if the navigation is correct, an aircraft may deviate from the nominal route due to wind, for example. This error is called flight technical error (FTE). Here, the lateral-PDF due to FTE is assumed to follow normal distribution with a $\mathrm{SD}$ of $\sigma_{3}=1.28 \mathrm{NM}$.

4) Operational error

In addition to the above factors, the aircraft may deviate 
due to pilot or ATC errors, referred to here as operational error. Here, the lateral-PDF due to operational error is assumed to follow Laplace distribution (i.e., double exponential distribution) with the shape parameter of $\lambda=15 \mathrm{NM}$ (= SD of 10.61 NM). The operational error is assumed to happen with a probability of $c=2.76 \times 10^{-7}$. However, the source of this value is not described.

\section{5) Lateral-PDF}

Finally, the total lateral-PDF is obtained using the following formula. With this lateral-PDF, the collision probability satisfies the TLS:

$$
\begin{aligned}
f(y)= & (1-c)\left\{(1-b) N\left(y ; 0, \sigma_{1}{ }^{2}+\sigma_{3}{ }^{2}\right)\right. \\
& \left.+b N\left(y ; 0, \sigma_{2}{ }^{2}+\sigma_{3}{ }^{2}\right)\right\}+c L(y ; 0, \lambda)
\end{aligned}
$$

where

$$
N\left(y ; 0, \sigma^{2}\right)=\frac{1}{\sqrt{2 \pi \sigma}} \exp \left(-y^{2} / 2 \sigma^{2}\right)
$$

and

$$
L(y ; 0, \lambda)=\frac{1}{2 \lambda} \exp (-|y| / \lambda) .
$$

\subsection{ASEPS model}

While the Reich model is a stationary model, the ASEPS model is a time-dependent model, and therefore a more accurate evaluation is expected. The ASEPS and Reich models are similar in many aspects, but ASEPS essentially replaces an arbitrary assumption for a lateral position distribution with one based on the probability of an aircraft reaching the adjacent track given a distribution of deviation angles and distribution of intervention times. The main factor of large deviation is known to be an operational error, and other deviation factors are considered typical deviations. In this model, the two routes are spaced by $S_{y}$, and the aircraft is assumed to start deviating with the constant angle $\theta$ at $t=t_{d}$, and this deviation ends at $t=t_{3}$, as shown in Fig. 2 . Here, $t_{3}-t_{d}$ indicates the total response time to resolve the conflict, and this value together with $\theta$ affect the magnitude of deviation. This consideration is a benefit of the time-dependent model to estimate collision probability more accurately. The surveillance system alerts the deviation when the deviation becomes greater than a certain distance (alerting distance). The ground speed on each route is assumed to be always constant at $V_{1}$ and $V_{2}$, respectively.

The detailed derivation is not explained in detail here, but the following are the key parameters of the model.

1) ATC intervention time

After receiving the first deviation report, ATC needs to understand the situation and fully resolve the conflict for $\tau_{i}$ after the first report at $t=t_{2}$. Therefore, the ATC intervention time $\left(\tau_{i}\right)$ is defined as $t_{3}-t_{2}$. The larger this parameter is, the higher the collision probability.

2) Distribution of deviation angle

The deviation angle $(\theta)$ is also a key parameter, and the larger this parameter is, the higher the collision probability. Here, the following PDF of the deviation angle is used. This function is a truncated exponential distribution, and $\theta$ is de-

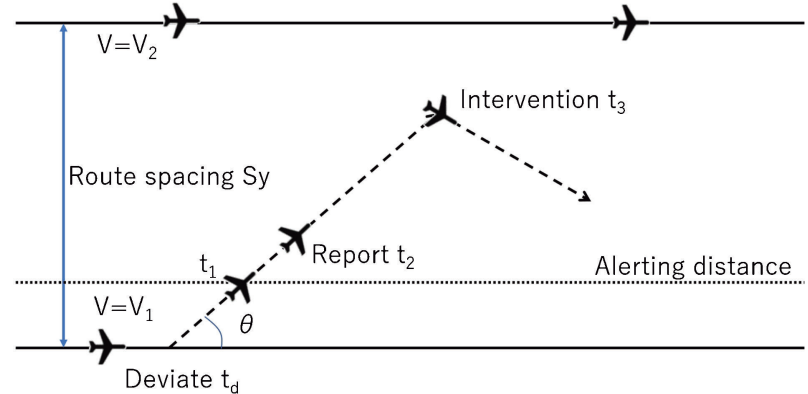

Fig. 2. Deviation scenario. The aircraft starts deviating at $t=t_{d}$ with a deviation angle of $\theta$. The aircraft reaches the deviation distance at $t=t_{1}$, and the first report after reaching the deviation distance is at $t=t_{2}$. ATC manages the final safe intervention at $t=t_{3}$.

fined $[0 \pi / 2]$ :

$$
f_{\theta}(\theta)=\frac{\exp (-\theta / a)}{a(1-\exp (-\pi / 2 a))}
$$

\section{3) Occupancy}

The occupancy $(E)$ is twice the number of proximate pairs observed at a sampling point divided by the total number of aircraft that passed the sampling point. In other words, this value reflects the probability that the aircraft horizontally collide with another aircraft when the aircraft crosses an adjacent route.

4) Collision probability

Finally, the collision probability (CP) is calculated using the following formula. The parameters are summarized in Table 1.

$$
\begin{aligned}
C P= & 2 \Omega \frac{E \lambda_{x}}{d_{x}} P_{z} \\
& \times \iint_{V_{1}, V_{2}} \int_{\delta_{s}=-\sigma_{T}}^{\sigma_{T}} \Gamma\left(V_{1}, V_{2}, T_{p}, \alpha^{*}, \tau^{*}\right) \\
& \times f_{V}\left(V_{1}\right) f_{V}\left(V_{2}\right) f_{s}\left(\delta_{s}\right) d V_{1} d V_{2} d \delta_{s}
\end{aligned}
$$

$$
\begin{gathered}
\Gamma\left(V_{1}, V_{2}, T_{p}, \alpha^{*}, \tau^{*}\right) \\
=\frac{1}{T_{p}} \sum_{i} \alpha_{i} \int_{T_{a}=\tau_{i}+T_{L}}^{\tau_{i}+T_{L}+T_{p}} \int_{\theta=\theta^{*}}^{\pi / 2} f_{\theta}(\theta)\left(1+\frac{\left|V_{1} \cos \theta-V_{2}\right|}{V_{1} \sin \theta}\right) \\
\quad \times\left(1+\frac{\pi \lambda_{x}}{2 \sqrt{V_{1}^{2}+V_{2}^{2}-2 V_{1} V_{2} \cos \theta}} \frac{2 \lambda_{z}}{\dot{z}}\right) d \theta d T_{a} \\
\sin \theta^{*}=\frac{S_{y}-d_{s}-\delta_{s}}{T_{a} V_{1}}
\end{gathered}
$$

Several associated parameters are defined as distributions, and the average collision probability is calculated with the integral of functions. In Eq. (7), $\theta^{*}$ indicates the minimum deviation angle where the deviating aircraft laterally reaches the adjacent route. Therefore, only an angle greater than $\theta^{*}$ should be considered for the collision. 
Table 1. Parameters list.

\begin{tabular}{|c|c|c|c|}
\hline Parameters & Descriptions & Typical values & Value source \\
\hline$\Omega$ & Probability that the aircraft makes a large deviation & $7 \times 10^{-5}$ & North Atlantic historical data ${ }^{12)}$ \\
\hline$a$ & Parameter of deviation angle distribution & 16 degrees & North Atlantic historical data \\
\hline$E$ & Occupancy & 1.1 & Large value for most airspaces ${ }^{9)}$ \\
\hline$\lambda_{x}$ & Average aircraft length & $0.0399 \mathrm{NM}$ & B777-300ER \\
\hline$\lambda_{z}$ & Average aircraft height & $0.0100 \mathrm{NM}$ & B777-300ER \\
\hline$d_{x}$ & $\begin{array}{l}\text { Considered range of aircraft which may collide on } \\
\text { the adjacent route }\end{array}$ & $120 \mathrm{NM}$ & Arbitrary value \\
\hline$P_{z}$ & Vertical overlap probability $\left(=P_{z}(0)\right)$ & 0.55 & $\begin{array}{l}\text { Manual on Monitoring the Application of } \\
\text { Performance-based Horizontal Separation Minima }{ }^{13)}\end{array}$ \\
\hline$\sigma_{T}$ & $\begin{array}{l}\text { 1-sigma value of lateral deviation considering RNP } \\
\text { definition }\end{array}$ & RNP value/1.96 & - \\
\hline$f_{V}(V)$ & Distribution of ground speed (Laplace distribution) & Average: $480 \mathrm{kt}, \mathrm{SD}: 17 \mathrm{kt}$ & Barry ${ }^{9)}$ \\
\hline$f_{s}\left(\delta_{s}\right)$ & $\begin{array}{l}\text { Distribution of lateral RNP position variation } \\
\text { (Truncated normal distribution) }\end{array}$ & Average: $0, \mathrm{SD}: \sigma_{T}$ & Barry $^{9)}$ \\
\hline$T_{p}$ & Surveillance frequency & - & Depends on the surveillance system \\
\hline$T_{L}$ & Surveillance latency & - & Depends on the surveillance system \\
\hline$\tau_{i}, \alpha_{i}$ & Action time and its ratio to resolve the conflict & - & Depends on the considered scenario \\
\hline$\dot{z}$ & Relative vertical speed at time of collision & $1.5 \mathrm{kt}$ & $\begin{array}{l}\text { Manual on Monitoring the Application of } \\
\text { Performance-based Horizontal Separation Minima }{ }^{13)}\end{array}$ \\
\hline
\end{tabular}

\section{Application of ASEPS Model to RNP 2 under a Ra- dar Environment}

\subsection{Directions of safety assessment of lateral separation for RNP 2 under a radar environment}

The current 15-NM safety analysis does not consider the surveillance environment, so this 15-NM separation can be applied without radar surveillance. However, if the surveillance (i.e., time histories of aircraft position) is available, ATC can also obtain additional aircraft information and change instructions accordingly. Since the ASEPS model considers the surveillance environment, application of the ASEPS model to the proposed RNP2 operation can potentially reduce the separation standard. On the other hand, the assumptions used in the 15-NM safety analysis should be used for consistency as long as they are appropriate. Therefore, in this section, ASEPS model parameters are carefully determined considering the difference between the proposed RNP2 operation and the ASEPS model scenario/ 15-NM safety analysis.

\subsection{Determination of parameters in ASEPS model}

\section{1) $\lambda_{x}, \lambda_{z}, P_{z}(0), f_{V}(V), f_{s}\left(\delta_{s}\right)$}

The values of parameters $\lambda_{x}, \lambda_{z}, P_{z}(0), f_{V}(V)$, and $f_{s}\left(\delta_{s}\right)$ are set to typical values shown in Table 1 . These values are used in various safety assessments in ICAO, and these parameters are not situation-dependent.

2) $\Omega, a$

These parameters include the deviation probability and the deviation angle; however, such large deviations are rarely observed, so it is difficult to obtain statistical data. In the ASEPS model, these values are obtained based on historical data of the North Atlantic with 501 deviation data. ${ }^{12)}$ The author also previously investigated the parameters used here with the limited deviation data in Japan. ${ }^{14)}$ The results show that these parameters are more conservative (buffered) than the data obtained in Japan, so the same parameters are used here.
3) $T_{p}, T_{L}$

The ASEPS model was initially developed on oceanic routes, where surveillance has traditionally been poor, to improve standards for the case of improving surveillance by space-based ADS-B. On the other hand, this paper considers an area under radar coverage. On an en-route flight, air route surveillance radar (ARSR) updates the aircraft position every $10 \mathrm{~s}$ with little latency. Therefore, $T_{p}=10[\mathrm{~s}]$ and $T_{L}=0$ are used in this analysis.

4) Alerting distance

The alerting distance is an arbitrary value, but longer separation is required if it is set large. However, if it is set too small, false alerting may occur frequently. This time, the alerting distance is set to $1 \mathrm{NM}$.

5) $\tau_{i}, \alpha_{i}$

These parameters affect the time to resolve the conflict. However, it is difficult to use the same values as the ASEPS model because the ASEPS model was intended to be developed for oceanic flights. In the ASEPS model, surveillance is assumed to be provided by space-based ADS-B, and communication is via CPDLC data transmission or HF voice communication. On the other hand, on en-route flights under radar coverage, surveillance is provided by ARSR and communication is via VHF voice communication. Since careful consideration is required, the values are determined in Sec. 3.3.

6) $E$

As for occupancy, although 1.1 is a sufficiently large value, the actual value can be obtained using the current traffic data. Therefore, the occupancy is calculated using the historical data in Japanese airspace. A detailed calculation is given in Sec. 3.4.

\subsection{Determination of action time}

The ASEPS model has been a subject of long discussions and various types of action time have been considered. Even though it focuses on oceanic flight operations, the general process for the action time should be the same. Table 2 shows the action time under normal operations discussed 
Table 2. Action time during normal operations.

\begin{tabular}{lc}
\hline \multicolumn{1}{c}{ Required actions } & Action time $[\mathrm{s}]$ \\
\hline Screen update time/controller & 30 \\
conflict recognition & 15 \\
Controller message composition & 90 \\
CPDLC uplink & 30 \\
Pilot reaction & 75 \\
Aircraft inertia and climb & 240 \\
Total & \\
\hline
\end{tabular}

in Manual on Monitoring the Application of Performancebased Horizontal Separation Minima. ${ }^{13)}$

The required actions are listed, and each action time is set. In this example, the total action time is 240 s (i.e., 4 min). As well as normal operations, several possible other operations (e.g., failure of primary surveillance) are considered, and the action time is set. The probability of each operation occurrence $\left(=\alpha_{i}\right)$ is set, and the average collision probability is calculated as shown in Eq. (5).

In this example, surveillance is provided by ADS-C, and communication is provided by a CPDLC datalink. Both systems can fail, and these failure scenarios are considered. However, under a radar environment, surveillance is provided by air route surveillance radar (ARSR) and communication is VHF voice communication. Both systems are sufficiently reliable, and no alternative systems need to be considered.

Next, each action in Table 2 is investigated in more detail: 1) Screen update time and controller conflict recognition are also required under a radar environment. However, unlike CPDLC, 2) Controller message composition is not required for voice communication. CPDLC is usually sent via satellite connections, so a certain time-lag is observed. On the other hand, voice communication is real-time and requires the communication time only, which replaces 3) CPDLC uplink time. 4) Pilot reaction time is not required either, because the pilot understands the ATC instruction over the voice communication. 5) The aircraft inertia and climb are required in the proposed RNP2 operation, too. Therefore, three factors (i.e., controller conflict recognition, voice communication, and aircraft response time to resolve conflict) should be considered for the proposed RNP2 operations.

There have been several studies investigating the durations of the above actions. Cardosi et al. investigated the ATC reaction time after alerting, and reported the average to be $17.74 \mathrm{~s}^{15)}$ Nickelson investigated the time between ATC notification and start of aircraft trajectory change (Communication time), and reported its average to be $34.4 \mathrm{~s} .{ }^{16)} \mathrm{He}$ also investigated the completion time of trajectory change, and reported its average to be $39.4 \mathrm{~s}$. The sum of these three factors becomes the total action time. Therefore, these three factors are modeled by the distribution function, and the percentiles of these times are obtained through numerical simulations.

All three factors are modeled using log-normal distribution, which is an asymmetric distribution. The PDF of lognormal distribution is defined using the following formula:
Table 3. Percentile values of each action time.

\begin{tabular}{lc}
\hline & Values in references \\
\hline ATC reaction time & {$[9,10,16,27,30] \mathrm{s}$ for $[5,10,50,90,95]$} \\
& percentile $^{15)}$ \\
Communication time & {$[33.3,40.0,55.1,69.2] \mathrm{s}$ for $[50,75,95,99]$} \\
& percentile $^{16)}$ \\
Completion of & {$[35.7,49.0,75.8,109.3] \mathrm{s}$ for $[50,75,95,99]$} \\
trajectory change & percentile $^{16)}$ \\
\hline
\end{tabular}

Table 4. Estimated parameters.

\begin{tabular}{|c|c|c|c|c|c|}
\hline & \multicolumn{3}{|c|}{ Estimated parameters } & \multirow{2}{*}{$\begin{array}{c}\text { Estimated } \\
\text { average [s] }\end{array}$} & \multirow{2}{*}{$\begin{array}{c}\text { Actual } \\
\text { average }[s\end{array}$} \\
\hline & $t_{0}$ & $\mu$ & $\sigma$ & & \\
\hline ATC reaction time & 2.45 & 2.61 & 0.45 & 17.45 & 17.74 \\
\hline Communication time & 17.33 & 2.77 & 0.52 & 35.61 & 34.4 \\
\hline Trajectory change time & 0.0 & 3.58 & 0.46 & 39.80 & 39.4 \\
\hline
\end{tabular}

$$
g(x ; \mu, \sigma)=\frac{1}{\sqrt{2 \pi} \sigma x} \exp \left(-\frac{(\log (x)-\mu)^{2}}{2 \sigma^{2}}\right)
$$

This function is defined in $x>0$. There should be minimum time for each action time, so the following shifted function is used to model each action time:

$$
t_{0}+g\left(t-t_{0} ; \mu, \sigma\right)
$$

There are three parameters $\left(t_{0}, \mu\right.$, and $\left.\sigma\right)$ in this model. Each reference includes several percentile values as summarized in Table 3, so the parameters are optimized to minimize the mean squared error for available percentile values.

Table 4 summarizes the estimation results, and Fig. 3 shows the cumulative probability of each action time. Each estimated average is similar to the actual one, and the actual percentile values are well estimated by the obtained function.

Here, the trajectory change time is modified to fit actual data in Japan. This trajectory change time assumes the aircraft climb, and the trajectory change is completed when $1000 \mathrm{ft}$ altitude change is achieved. This also means that the ASEPS model assumes that the aircraft is exposed to the risk until altitude change is completed, including climbing as if the aircraft stays at the same flight level, which is a conservative assumption. One-thousand feet is sufficient vertical separation, but the vertical overlap probability becomes less than $2.0 \times 10^{-3}$ if the aircraft climbs only $300 \mathrm{ft} .{ }^{17}$ ) Here, $300 \mathrm{ft}$ is the required vertical deviation for contingency procedure, ${ }^{2)}$ which also avoids vertical overlap to some extent. Therefore, the author proposes that two separate action times are obtained to climb $300 \mathrm{ft}$ and $1000 \mathrm{ft}$, and the collision probability is calculated considering the action time and vertical overlap probability of each climb distance.

Japanese en-route flight data for five days in 2018 are used for verification. The aircraft that climb or descend above FL290 reduced vertical separation minima (RVSM) airspace by more than $1000 \mathrm{ft}$ are extracted, and the trajectory change time for $300 \mathrm{ft}$ climb/descent and $1000 \mathrm{ft}$ climb/descent are analyzed. A total of 2,447 aircraft climb data and 10,988 descent data are obtained. According to the pre-data analysis, the descent time is shorter than the climb time, so the climb time is used for a conservative calculation. Figure 4 and 


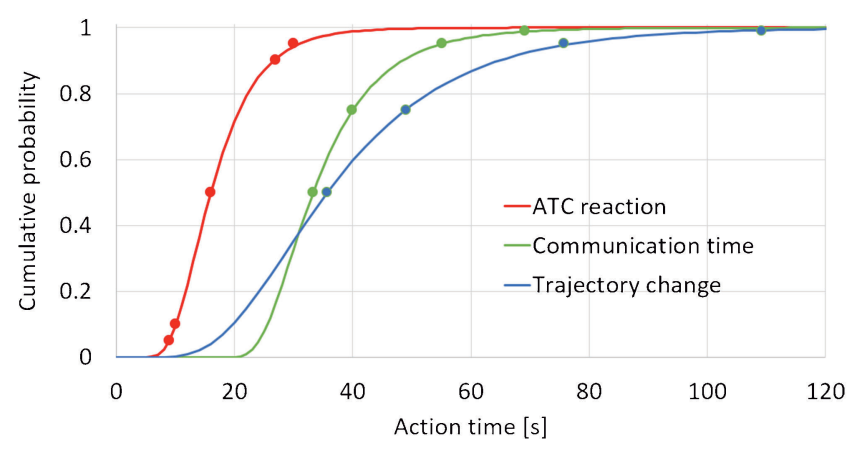

Fig. 3. Cumulative probability of each action time (Line: estimated distribution; Dot: actual percentile values).

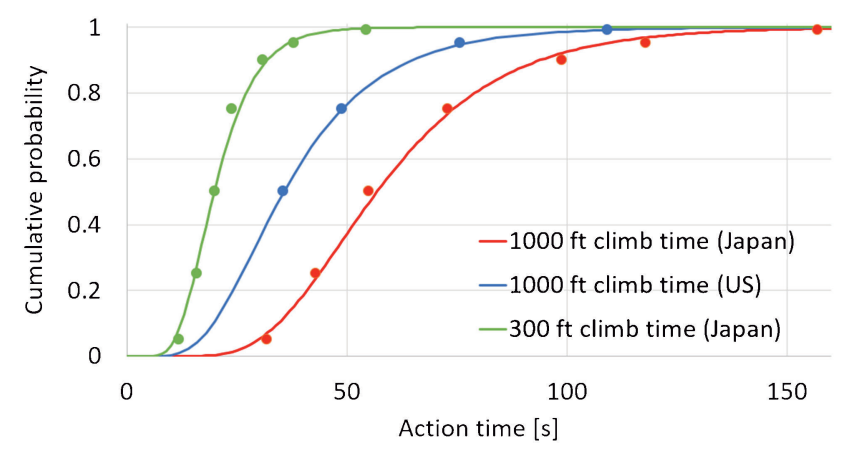

Fig. 4. Action time for trajectory change.

Table 5. Estimated parameters of trajectory change.

\begin{tabular}{|c|c|c|c|c|c|}
\hline & \multicolumn{3}{|c|}{ Estimated parameters } & \multirow{2}{*}{$\begin{array}{c}\text { Estimated } \\
\text { average [s] }\end{array}$} & \multirow{2}{*}{$\begin{array}{c}\text { Actual } \\
\text { average }[\mathrm{s}]\end{array}$} \\
\hline & $t_{0}$ & $\mu$ & $\sigma$ & & \\
\hline $1000 \mathrm{ft}$ climb (Japan) & 0.0 & 4.04 & 0.39 & 61.41 & 62.7 \\
\hline $1000 \mathrm{ft}$ climb (US) & 0.0 & 3.58 & 0.46 & 39.80 & 39.4 \\
\hline $300 \mathrm{ft}$ climb (Japan) & 0.0 & 2.99 & 0.37 & 21.29 & 22.4 \\
\hline
\end{tabular}

Table 5 show the action time of trajectory change and estimated results. The US data is the same as that used in Fig. 3. The results show that $1000 \mathrm{ft}$ climb time in Japan is longer than that in the US by more than $20 \mathrm{~s}$ on average. The reason is not identified, but it may be due to the cruise altitude used or the fleet mix in Japanese airspace, so the Japanese results will be used in this analysis.

When climbing $300 \mathrm{ft}$, the average action time is about $35 \%$ of that to reach $1000 \mathrm{ft}$. This means that the action time is almost linear to the change in altitude. The total action time can be also reduced if the trajectory is changed by only $300 \mathrm{ft}$.

Finally, the total action time is calculated based on three distributions (i.e., ATC reaction time, Communication time, and Trajectory change time). Assuming the three actions to be independent, the percentile of action time can be numerically calculated. The following values are obtained for the total action time:

[77, 89, 105, 135 and 165] s for [25, 50, 75, 95 and 99] percentiles assuming the trajectory change in US $1000 \mathrm{ft}$ climb;

[64, 72, 83, 102 and 119] s for [25, 50, 75, 95 and 99] percentiles assuming trajectory change in Japan $300 \mathrm{ft}$ climb; and
[95, 110, 129, 166 and 199] s for [25, 50, 75, 95 and 99] percentiles assuming trajectory change in Japan $1000 \mathrm{ft}$ climb.

Using the above results, $\tau_{i}$ and $\alpha_{i}$ are set to the following values. (Since $15-\mathrm{NM}$ safety analysis assumes a maximum action time of $3 \mathrm{~min}$, the same value is used here for the worst case scenario.)

$$
\begin{aligned}
{\left[\tau_{i}, \alpha_{i}\right]=} & {[77,0.25],[89,0.25],[105,0.25], } \\
& {[135,0.2],[165,0.04] \text { and }[300,0.01] } \\
& (\mathrm{US} 1000 \mathrm{ft} \text { climb); } \\
{\left[\tau_{i}, \alpha_{i}\right]=} & {[64,0.25],[72,0.25],[83,0.25], } \\
& {[102,0.2],[119,0.04] \text { and }[300,0.01] } \\
& (\text { Japan 300 ft climb); and } \\
{\left[\tau_{i}, \alpha_{i}\right]=} & {[95,0.25],[110,0.25],[129,0.25], } \\
& {[166,0.2],[199,0.04] \text { and }[300,0.01] } \\
& (\text { Japan 1000 ft climb). }
\end{aligned}
$$

If the data for Japan $300 \mathrm{ft}$ climb is used, the risk is underestimated because $300 \mathrm{ft}$ is not sufficient vertical separation. Therefore, the collision probability is calculated for a conservative estimation.

$$
\begin{aligned}
& C R\left(P_{z}=P_{z}(0)=0.55,\left[\tau_{i}, \alpha_{i}\right]: \text { Japan } 300 \mathrm{ft}\right) \\
& +C R\left(P_{z}=P_{z}(300)=2 \times 10^{-3},\left[\tau_{i}, \alpha_{i}\right]: \text { Japan } 1000 \mathrm{ft}\right)
\end{aligned}
$$

Aircraft are assumed to stay at the initial flight level when $300 \mathrm{ft}$ climb time is used, and aircraft are assumed to be separated vertically by $300 \mathrm{ft}$ when $1000 \mathrm{ft}$ climb time is used. The original calculation assumes the aircraft stays at the initial flight level until climbing by $1000 \mathrm{ft}$. The new calculation is still conservative, but a more realistic calculation is possible because $300 \mathrm{ft}$ climb time is also considered.

\subsection{Determination of occupancy}

Occupancy is defined as the average number of aircraft on an adjacent route that may collide with the aircraft under consideration. It is a function of $d_{x}$, but $d_{x}$ is also included in Eq. (5), so the calculated collision probability shows the same, or at least similar, results regardless of $d_{x}$. Here, $d_{x}=120 \mathrm{NM}$ is used as the other references use this value. Even if the traffic volume increases, occupancy does not necessarily increase. Occupancy becomes large when the traffic is equally distributed between two routes, when the traffic is observed at the same time on both routes, and when dense traffic is observed on both routes. Since occupancy does not necessarily depend on the traffic volume, it is difficult to estimate occupancy in the future. Therefore, it is reasonable to confirm that the occupancy calculated using the actual data is sufficiently smaller than the value used (here, namely 1.1).

Occupancy is calculated using the following formula:

$$
E=\frac{\sum_{j=1}^{m} \sum_{i=1}^{n_{j}} N_{i}^{j}}{\sum_{j=1}^{m} n_{j}}
$$

where $n_{j}$ indicates the number of aircraft on $j$ th route and $N_{i}^{j}$ indicates the number of aircraft in the proximity of aircraft $i$ on $j$ th route. Only aircraft flying at the same flight level are considered as aircraft in the proximity of a given aircraft. Occupancy becomes two when all aircraft are separated by only 


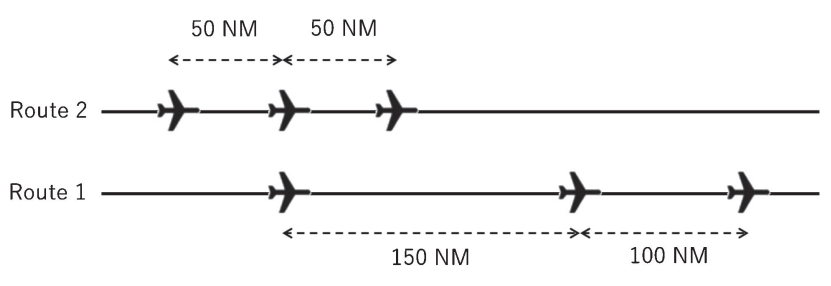

Fig. 5. Example of calculating occupancy.

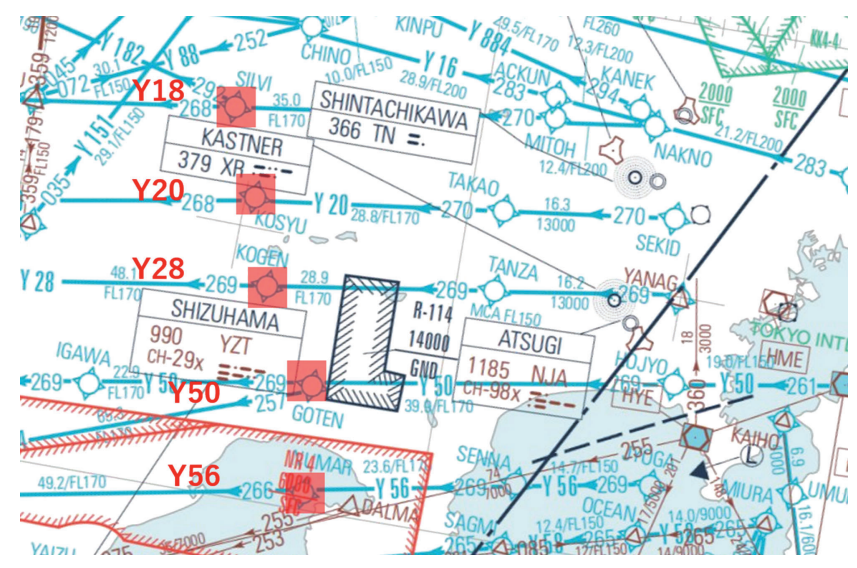

Fig. 6. Considered parallel routes (AIS Japan en-route chart).

$120 \mathrm{NM}$ on both routes. Figure 5 is used as an example to calculate route occupancy. In this example, occupancy can be calculated in the following way:

$$
\begin{aligned}
& n_{1}=3, \quad n_{2}=3, \quad N_{1}^{1}=3, \quad N_{2}^{1}=1, \quad N_{3}^{1}=0, \\
& N_{1}^{2}=1, \quad N_{2}^{2}=1, \quad N_{3}^{2}=2 \\
& E=\frac{3+1+0+1+1+2}{3+3}=1.333
\end{aligned}
$$

Note that the total occupancy of multiple routes does not become the sum or the average of each occupancy of two adjacent routes. In this way, occupancy is calculated using actual flight data. This time, one-year data of 2018 is used for the calculation. Figure 6 shows the en-route chart near Tokyo, and the busiest parallel routes in Japan where the aircraft go westbound from Tokyo. Occupancy is calculated at the waypoints of SILVI (Y18), KOSYU (Y20), KOGEN (Y28), GOTEN (Y50), and NUMAR (Y56). Although the waypoints are not exactly located at along-track positions, this hardly affects the calculation result. The results show that the total occupancy is 0.650 , which is sufficiently below 1.1. To investigate the relationship between the traffic volume and occupancy, occupancy is calculated each day, and the relationship between occupancy and traffic volume is shown in Fig. 7. This figure does not show a clear relationship between occupancy and traffic volume. Among the route combinations, Y20-Y28 shows the highest occupancy, but the traffic volume is third among the four route combinations. The total occupancy is greater than that of each route combination because Y20, Y28 and Y50 have parallel routes on both sides, which increases the occupancy. Although the daily distribution of occupancy is also high, none of the days

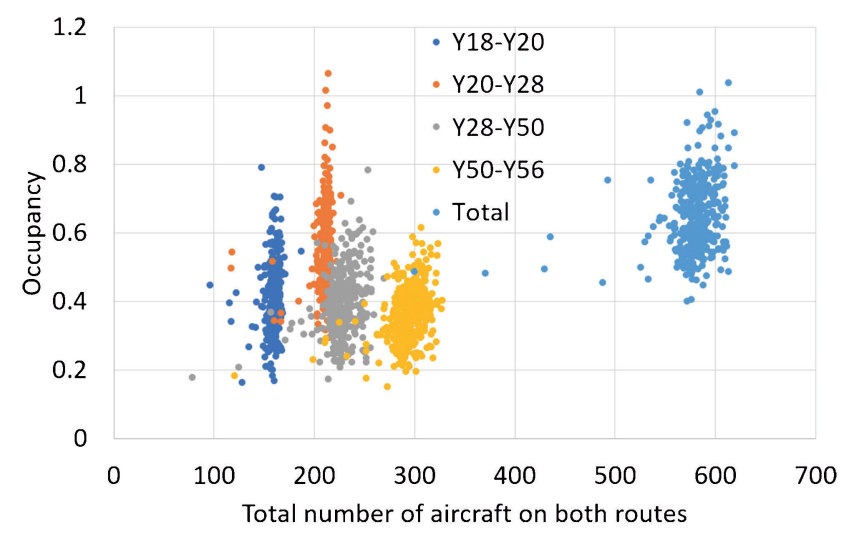

Fig. 7. Relationship between occupancy and traffic volume among route combinations.

have an occupancy greater than 1.1. Considering this fact, $E=1.1$ is sufficiently conservative, and this value is used for the calculation.

\subsection{Additional factors considered}

Using the above values, the collision probability can be calculated. However, to keep consistency, it is better to consider the same factors as those applied for 15-NM safety analysis. In this analysis, as explained in Sec. 2.2, four factors (i.e., 1) GNSS navigation error, 2) Navigation error during a RAIM outage, 3) Flight technical error, and 4) Operational error) are considered. GNSS navigation error and flight technical error are included in RNP position variation $\left(f_{s}\left(\delta_{s}\right)\right)$. Operational error is also modeled. However, navigation error during a RAIM outage is not explicitly modeled. RAIM outage is one of the GNSS problems, and integrity cannot be monitored under RAIM outage. RAIM outage prediction is given before the flight, so in general, the aircraft does not encounter RAIM outage. On the other hand, GNSS loss may be more critical for RNP 2 flights. Since DME/ DME updating is not allowed for RNP 2 flights, RNP 2 requirements cannot be met once GNSS is lost. Therefore, GNSS loss is considered instead of RAIM outage here to cover various GNSS problems. Even if GNSS loss occurs, the aircraft can roughly follow the route by keeping the current heading or track angle. In the 15-NM safety analysis, 9deg track angle deviation with a $95 \%$ probability (i.e., $\mathrm{SD}=$ $9 / 1.96=4.59 \mathrm{deg}$ ) is assumed for RAIM outage, which may be reasonable for a GNSS loss scenario. Here, the deviation angle is assumed to follow the exponential distribution shown in Eq. (4), with $\mathrm{SD}=4.59 \mathrm{deg}$. The SD of Eq. (4) is easily calculated using the following formula:

$$
\begin{aligned}
\mathrm{SD}^{2}=E\left(\theta^{2}\right)-E(\theta)^{2} & \int_{0}^{\pi / 2} \theta^{2} f_{\theta}(\theta) d \theta \\
= & \left(2 E(\theta)-\frac{\pi^{2}}{4} \exp \left(-\frac{\pi}{2 a}\right)\right) a^{2}\left(1-\exp \left(-\frac{\pi}{2 a}\right)\right)
\end{aligned}
$$




$$
\begin{aligned}
E(\theta) & =\int_{0}^{\pi / 2} \theta f_{\theta}(\theta) d \theta \\
& =\left(\exp \left(-\frac{\pi}{2 a}\right)\left(-\frac{\pi}{2}-a\right)+a\right) a^{2}\left(1-\exp \left(-\frac{\pi}{2 a}\right)\right)
\end{aligned}
$$

When SD is $4.59 \mathrm{deg}, a$ becomes $4.59 \mathrm{deg}$. The standard deviation is close to the parameter $a$ as expected, since the distribution is mostly exponential: truncation at $90 \mathrm{deg}$ will have little effect with such a small scale parameter.

In addition, GNSS loss frequency $(\Omega)$ should be determined. In the 15-NM safety assessment, 10-min RAIM outage for one-year flight is assumed. GNSS loss is a very rare event, and it will happen less often than RAIM outage. However, here, GNSS loss is assumed to happen as the same frequency of RAIM outage for a conservative estimation. Additionally, $\Omega$ is defined as the number of deviations per flight hour, not the time ratio. Here, RAIM outage is assumed to happen for $10 \mathrm{~min}$ once in a year, so another GNSS loss is assumed to happen at the same rate (i.e., once a year). Finally, $\Omega$ is set using the following formula assuming twice-a-year GNSS loss:

$$
\Omega=\frac{2}{24 \times 365.25}=2.28 \times 10^{-4}
$$

This value is more than 10 times larger than the frequency of operational error. It may be too large but can be used for a conservative estimation. The other parameters are set the same as explained in previous sections because ATC reaction does not change depending on the reason for deviation. Therefore, using the values of $a$ and $\Omega$ defined in this section, the collision probability is calculated again for a GNSS loss scenario. The sum of the collision probability for operational error and GNSS loss becomes the total collision probability.

\subsection{Calculation results}

Figure 8 shows the calculation results. As seen in the figure, the collision probability is below TLS when lateral separation is $8 \mathrm{NM}$ or greater when using the proposed calculation method. If the trajectory change time obtained from the US is used directly, 10-NM separation is needed. This means that the proposed method succeeds in reducing the estimated collision probability.

Figure 9 shows the ratios of collision probability in each factor using the proposed method. As seen in the figure, the operational error (i.e., $300 \mathrm{ft}$ ) occupies most of the collision probability (about 95\%). The next factor is the collision probability by GNSS loss (i.e., $300 \mathrm{ft}$ ), and about $4 \%$ is occupied. The rest (i.e., operational error and GNSS loss $1000 \mathrm{ft}$ ) occupy less than $1 \%$ of the total collision probability. As seen in Table 5, the action time difference between $300 \mathrm{ft}$ and $1000 \mathrm{ft}$ is about $40 \mathrm{~s}$, which almost doubles the collision probability. However, when $1000 \mathrm{ft}$ climb time is used, aircraft are assumed to be already separated by $300 \mathrm{ft}$, reducing the vertical overlap probability by $275\left(=0.55 /\left(2 \times 10^{-3}\right)\right)$ times. Therefore, the collision probability when the aircraft are vertically separated by more than $300 \mathrm{ft}$ becomes almost

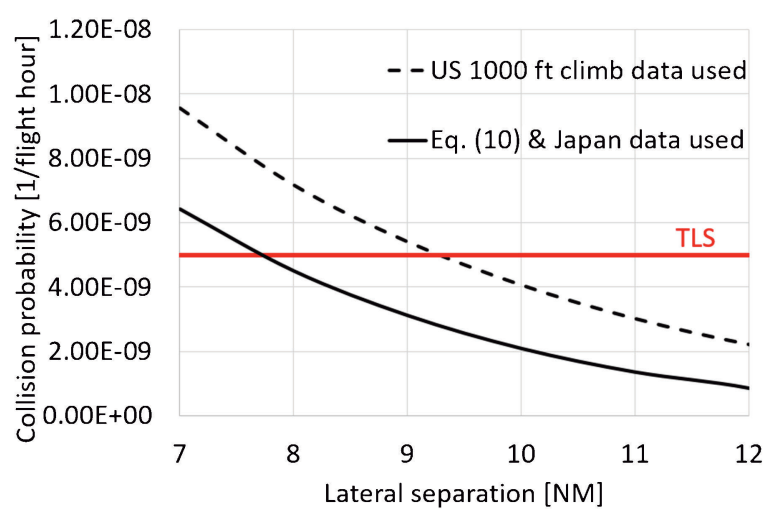

Fig. 8. Collision probability for each lateral separation. (US data uses $1000 \mathrm{ft}$ climb data only. Japanese data uses both $300 \mathrm{ft}$ and $1000 \mathrm{ft} \mathrm{climb}$ data with Eq. (10).)

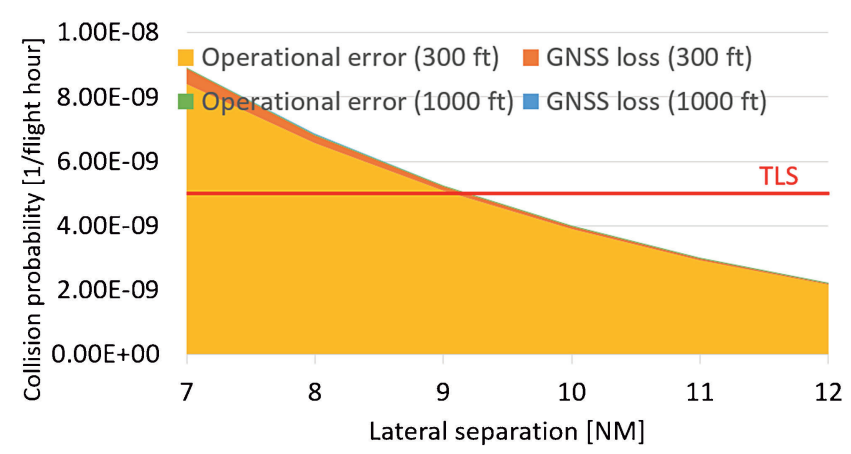

Fig. 9. Ratios of collision probability in each factor.

negligible.

According to this result, 8-NM lateral route spacing can be introduced safely. However, this calculation still uses several conservative assumptions. For example, the occupancy of 1.1 is used in this calculation, while the actual value for occupancy is 0.650 in Japanese airspace. The aircraft size used in this calculation is also larger than average. Therefore, it may be possible to reduce the possible lateral route spacing further; although it is also important to choose the parameters very carefully.

\section{Conclusion}

This paper considered the safety assessment of route spacing for RNP 2 under radar coverage. Although a 15-NM separation standard is established in the current criteria, this safety assessment did not consider a surveillance environment. Therefore, the author used the latest collision risk model that considers a surveillance environment. Since this model was intended to be used for oceanic route systems, this paper modified the model and parameters by maintaining consistency with old criteria and actual data analysis. To estimate the collision probability more accurately, trajectory change times to $300 \mathrm{ft}$ and $1000 \mathrm{ft}$ were considered separately. According to the calculation results, 8-NM route spacing is possible, while $15 \mathrm{NM}$ is the current criteria. This does not necessarily mean that 8 -NM route spacing is the possible minimum distance, and this possible route spacing changes depending on the parameters chosen. 


\section{References}

1) International Civil Aviation Organization: Performance-based Navigation (PBN) Manual, Doc 9613 Fourth edition, 2013.

2) International Civil Aviation Organization: Procedures for Air Navigation Services - Air Traffic Management (PANS-ATM), Doc 4444 Sixteenth edition, 2016.

3) International Civil Aviation Organization: Regional Supplementary Procedures, Doc 7030 Fifth edition, 2008.

4) Reich, P. G.: Analysis of Long-range Air Traffic Systems: Separation Standards - I, J. Navigation, 19, 1 (1966), pp. 88-98.

5) Reich, P. G.: Analysis of Long-range Air Traffic Systems: Separation Standards - III, J. Navigation, 19, 3 (1966), pp. 331-347.

6) International Civil Aviation Organization: Methodology for the Derivation of Separation Minima Applied to the Spacing between Parallel Tracks in ATS Route Structures, Cir 120 Second edition, 1976.

7) International Civil Aviation Organization, Guidelines for the Implementation of Lateral Separation Minima, Cir 341-AN/184, 2015.

8) Barry, S. and Aldis, G.: A Model for Lateral Separation Standards Including Surveillance and Communication, ICAO SASP-WG/25, $\mathrm{WP} / 12,2014$

9) Barry, S.: Manual for ATS Surveillance Separation Using RCP240 Communications: Chapter 3-Mathematical Modelling, ICAO SASP-WG/33, WP/02, 2019.

10) Werner, K., Bredemeyer, J., and Delovski, T.: ADS-B over Satellite:
Global Air Traffic Surveillance from Space, 2014 Tyrrhenian International Workshop on Digital Communications - Enhanced Surveillance of Aircraft and Vehicles, 2014.

11) Aldis, G.: Lateral Separation of GNSS Aircraft Based on a $7 \mathrm{NM}$ Navigational Tolerance, ICAO SASP/WG/WHL/13, WP/49, 2008.

12) Thurlow, S. and Friedrichs, P.: Assessment of Reported Lateral Angular Deviations in the North Atlantic, ICAO SASP-WG/27, WP/13, 2015 .

13) International Civil Aviation Organization: Manual on Monitoring the Application of Performance-based Horizontal Separation Minima, Doc 10063, 2017.

14) Mori, R.: Lateral Deviation and Occupancy in Fukuoka FIR, ICAO SASP/1, IP/11, 2016.

15) Cardosi, K. M., Buerki-Cohen, J., Boole, P. W., Hourihan, J., and Mengert, P.: Controller Response to Conflict Resolution Advisory, DOT-VNTSC-FAA-92-14, DOT-FAA-NA-92-2, 1992.

16) Nickelson, M.: Aircraft Response Latencies to ATC Clearances, ICAO $\mathrm{SASP} / 2, \mathrm{WP} / 28,2018$.

17) International Civil Aviation Organization: Manual on a $300 \mathrm{~m}$ (1 000 ft) Vertical Separation Minimum between FL 290 and FL 410 Inclusive, Doc 9574-AN/934, Third edition, 2012.

Naoki Matayoshi Associate Editor 